\title{
ON CONJUGATE SPACES OF NAKANO SPACES
}

\author{
BY \\ SADAYUKI YAMAMURO
}

The Nakano space is a normed space. The relations between the modular and its norms are the most important problem in the theory of Nakano spaces. To determine the types of modulars whose norms are proportional has been completely solved by Amemiya [1] and Yamamuro [1].

The purpose of this paper is to consider the relations from another point of view, namely, as the relations between Young's inequality and Hölder's inequality. It is well known that the fundamental inequality in the theory of normed linear spaces is that of Hölder, and, if we consider the conjugate spaces of them, it is important to know when the Hölder's inequality takes equality sign. On the other hand, in the theory of Nakano spaces, the fundamental inequality is that of Young. Therefore, it is natural to study the relations between values at which the inequalities take equality signs.

This paper consists of three sections and two appendices. In $\$ 1$, as preparation to the following sections, we will restate without proofs some results of Nakano [1]. In $\$ 2$, we will show the equivalence of the reflexivity of the modular and that of its norms. Concerning the reflexivity of norms, Mori-Amemiya-Nakano's theorem [1] is fundamental and its proof is simple. At first, by using this theorem, we will give a new and simple proof of $\mathrm{Na}$ kano's reflexivity theorem of modular. Next, we will write a method by which we can prove the reflexivity of norms by that of modular. This method has been known among the group conducted by Professor Nakano, but has not yet been published. In $\$ 3$, we will calculate values of norms of a linear functional which plays important roles in the theory of Nakano spaces. In connection with the linear functional, we will give more information in Appendix 1. In Appendix 2, we will consider strict convexity of modulars and their norms.

\section{Nakano spaces and their conjugate spaces}

1.1. Semi-ordered linear spaces. Let $R$ be a vector lattice in the sense of Birkhoff [1]. Throughout this paper, we assume $R$ is a universally continuous semi-ordered linear space, namely, for any system of positive elements $x_{\lambda}(\lambda \in \Lambda)$ there exists the "infimum" $\cap_{\lambda \in \Lambda} x_{\lambda}$. The following definitions are well known:

$$
\begin{gathered}
x^{+}=x \cup 0 ; \quad x^{-}=(-x)^{+} ; \quad|x|=x^{+}+x^{-} \\
x \perp y \quad \text { if } \quad|x| \cap|y|=0 .
\end{gathered}
$$

Received by the editors April 25, 1957. 
For a sequence $x_{\nu}(\nu=1,2, \cdots)$, if $0 \leqq x_{1} \leqq x_{2} \leqq \cdots$ and $\bigcup_{\nu=1}^{\infty} x_{\nu}=x$ then we write $0 \leqq x_{\nu} \uparrow_{\nu=1}^{\infty} x$, and if $x_{1} \geqq x_{2} \geqq \cdots$ and $\bigcap_{l=1}^{\infty} x_{\nu}=x$, then we write $x_{\nu} \downarrow_{\nu=1}^{\infty} x$. A sequence $x_{\nu}(\nu=1,2, \cdots)$ is said to be order-convergent to $x$, if there exists a sequence $y_{\nu}(\nu=1,2, \cdots)$ such that $y_{\nu} \downarrow_{\nu=1}^{\infty} 0$ and $\left|x_{\nu}-x\right|$ $\leqq y_{\nu}(\nu=1,2, \cdots)$.

For an element $p \in R$, we define a projector $[p]$ as

$$
[p] x=\bigcup_{\nu=1}^{\infty}(x \cap \nu|p|) \quad \text { for every } x \geqq 0,
$$

and $[p] x=[p] x^{+}-[p] x^{-}(x \in R)$. This is a linear and idempotent operator on $R$ into $R$ and $|[p] x| \leqq|x|(x \in R)$.

A set $\mathfrak{B}$ of projectors is called an ideal, if (1) $\mathfrak{B} \bar{\ni}[0]$; (2) If $[p] \in \mathfrak{B}$ and $[p] \leqq[q]$, then $[q] \in \mathfrak{B}$; (3) If $[p] \in \mathfrak{B},[q] \in \mathfrak{B}$, then $[p][q] \in \mathfrak{B}$. An ideal is said to be maximal, if there exists no ideal which contains $\mathfrak{B}$ as a proper subset. We use $\mathfrak{p}, \mathfrak{q}, \cdots$ to denote maximal ideals.

The set of all maximal ideals on $R$ is denoted by $\mathfrak{F}$. For any $[p]$, we put

$$
U_{[p]}=\{p \in \mathfrak{F}:[p] \in p\} .
$$

Then, by the neighborhood system $\left\{U_{[p]}\right\}, \mathfrak{F}$ becomes a locally compact Hausdorff space and each $U_{[p]}$ is open and closed.

For two elements $x$ and $a$, we put

$$
A_{\lambda}=U_{\left[(\lambda a-x)^{+}\right]\left[a^{+}\right]}+U_{\left[(\lambda a-x)^{-}\right]\left[a^{-}\right]} \quad(-\infty<\lambda<\infty) .
$$

By this system of sets, we define

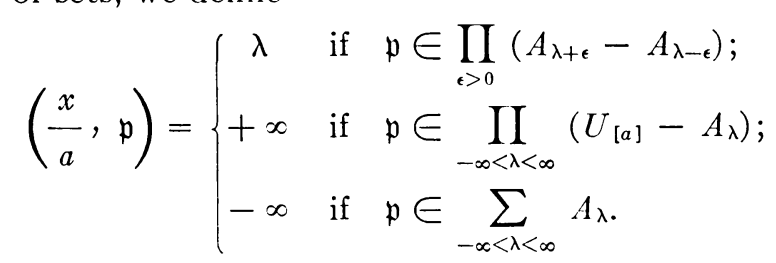

This function $(x / a, \mathfrak{p})$ is defined in $U_{[a]}$ and almost finite (namely, finite in an open set which is dense in $\left.U_{[a]}\right)$ and continuous in the extended sense. Moreover we have

$$
\begin{aligned}
\left(\frac{\alpha x+\beta y}{a}, \mathfrak{p}\right) & =\alpha\left(\frac{x}{a}, \mathfrak{p}\right)+\beta\left(\frac{y}{a}, \mathfrak{p}\right) ; \\
\left(\frac{x \cup y}{a}, \mathfrak{p}\right) & =\operatorname{Max} .\left\{\left(\frac{x}{a}, \mathfrak{p}\right),\left(\frac{y}{a}, \mathfrak{p}\right)\right\} ; \\
\left(\frac{x \cap y}{a}, \mathfrak{p}\right) & =\operatorname{Min} .\left\{\left(\frac{x}{a}, \mathfrak{p}\right),\left(\frac{y}{a}, \mathfrak{p}\right)\right\},
\end{aligned}
$$

if the right hand sides have sense. 
If $\phi(\mathfrak{p})$ is an almost finite, continuous function on $U_{[a]}$, there exists a sequence of projectors $\left[p_{\nu}\right] \uparrow_{\nu=1}^{\infty}[a]$ such that $\phi(p)$ is bounded in $U_{\left[p_{\nu}\right]}$ $(\nu=1,2, \cdots)$. The integral

$$
\int_{\left[p_{v}\right]} \phi(\mathfrak{p}) d \mathfrak{p} a
$$

is defined by the limit of the following partial sum:

$$
\sum_{\nu=1}^{\kappa} \phi\left(\mathfrak{p}_{\mu}\right)\left[p_{p, \mu}\right] a
$$

where $\mathfrak{p}_{\mu} \in U_{\left[p_{\nu, \mu}\right]}, \sum_{\mu=1}^{\kappa} U_{\left[p_{\nu, \mu}\right]}=U_{\left[p_{\nu}\right]}$ and

$$
|\phi(\mathfrak{p})-\phi(\mathfrak{q})|<\epsilon \quad\left(\mathfrak{p}, \mathfrak{q} \in U_{\left[p_{\nu}, \mu\right]}\right) .
$$

For this sequence of integrals, if there exists

$$
\lim _{\nu \rightarrow \infty} \int_{\left[p_{\nu}\right]} \phi(\mathfrak{p}) d \mathfrak{p} a,
$$

then the limit is denoted by

$$
\int_{[a]} \phi(\mathfrak{p}) d \mathfrak{p} a
$$

Nakano's spectral theory asserts that

$$
[a] x=\int_{[a]}\left(\frac{x}{a}, \mathfrak{p}\right) d \mathfrak{p} a,
$$

and, moreover, if

$$
x=\int_{[a]} \phi(\mathfrak{p}) d \mathfrak{p} a
$$

for some function $\phi(p)$ on $U_{[a]}$, then

$$
\phi(p)=\left(\frac{x}{a}, \mathfrak{p}\right) \quad\left(\mathfrak{p} \in U_{[a][x]}\right) .
$$

1.2. Nakano spaces. A real-valued function $m(x)$ on $R$ is said to be a modular on $R$ if it satisfies the following conditions: (1) $0 \leqq m(x) \leqq+\infty$; (2) For any $x$ there exist numbers $\alpha, \beta>0$ such that $m(\alpha x)>0$ and $m(\beta x)<\infty$; (3) For $\alpha, \beta>0$, we have $m((\alpha+\beta) / 2 x) \leqq\{m(\alpha x)+m(\beta x)\} / 2$; (4) If $|x| \leqq|y|$, then $m(x) \leqq m(y)$; (5) If $x \perp y$, then $m(x+y)=m(x)+m(y)$; (6) If $0 \leqq x_{\lambda} \uparrow_{\lambda \in \Delta} x$, $m(x)=\sup _{\lambda \in \Lambda} m\left(x_{\lambda}\right)$. We call such a space a Nakano space. Namely, the Nakano space is a universally continuous semi-ordered linear space on which a modular is defined. 
It is clear that so-called Orlicz space $L_{\Phi}$ is a Nakano space with the modu$\operatorname{lar}$

$$
m(x)=\int_{E} \Phi(|x(t)|) d t
$$

Another important example of Nakano space is $L_{p(t)}$, which is a set of measurable functions $x(t)(0 \leqq t \leqq 1)$ for which there exists a number $\alpha>0$ such that

$$
m(\alpha x)=\int_{0}^{1}|\alpha x(t)|^{p(t)} d t<+\infty .
$$

Here $p(t)$ must be a measurable function such that $p(t) \geqq 1(0 \leqq t \leqq 1)$.

Let $R$ be a Nakano space. We can define two kinds of norms:

$$
\begin{aligned}
& \text { the first norm: } \quad\|x\|=\inf _{\xi>0} \frac{1+m(\xi x)}{\xi} ; \\
& \text { the second norm: }\|\| x\|\|=\inf _{m(\xi x) \leqq 1} \frac{1}{|\xi|} .
\end{aligned}
$$

These formulae have been given by Professor Amemiya. It is easy to show that

$$
\|x\||\leqq\|x\| \leqq 2\|\mid\| x \| .
$$

An element $a$ is said to be simple, if $m(a)<+\infty$ and $m([p] a)=0$ implies $[p] a=0$. If $m(x)>0$ for every $x \neq 0$, then $m$ is said to be simple. If $m(\alpha x)$ $<+\infty$ for every $\alpha>0, x$ is called a finite element. If every element is finite, $m$ is said to be finite.

Let $\mu([p])$ be a function of projectors. We write

$$
\lim _{[p] \rightarrow \mathfrak{p}} \mu([p])=\alpha,
$$

if, for any $\epsilon>0$ we can find a projector $[p]$ such that

$$
|\mu([q])-\alpha|<\epsilon
$$

for every $[q] \leqq[p]$ such that $\mathfrak{p} \in U_{[q]}$. Nakano's spectral theory for modulars asserts that the function

$$
\omega(x \mid a, \mathfrak{p})=\lim _{[p] \rightarrow \mathfrak{p}} \frac{m([p] x)}{m([p] a)}
$$

is well defined for $p \in U_{[a]}$ and it is an almost finite, continuous function. Moreover, if we consider $m([p] a)$ as a measure,

$$
m([p] x)=\int_{[p]} \omega(x \mid a, \mathfrak{p}) m(d \mathfrak{p} a) .
$$


$\omega(\xi a / a, \mathfrak{p})$ is denoted by $\omega(\xi, a, \mathfrak{p})$.

1.3. Conjugate spaces of Nakano spaces. Let $\phi(x)$ be a linear (additive and homogeneous) functional on $R . \phi$ is said to be universally continuous if $x_{\lambda} \downarrow_{\lambda \in \Delta} 0$ implies $\inf _{\lambda \in \Lambda}\left|\phi\left(x_{\lambda}\right)\right|=0$. The set of all such functionals are called the conjugate space of $R$ and denoted by $\bar{R}$, which is also a universally continuous semi-ordered linear space, if we define $\phi \geqq 0$ as $\phi(x) \geqq 0$ for every $x \geqq 0$.

Since the Nakano space is a normed space, we can consider norm bounded functional. It is easy to see that a linear functional $\phi$ is norm bounded if and only if

$$
\phi(x) \leqq \gamma+\gamma m(x) \quad(x \in R)
$$

for some $\gamma>0$.

The set of all norm bounded linear functionals $\bar{x}$ of the conjugate space $\bar{R}$ is called the modular conjugate space and denoted by $\bar{R}^{m}$. If $m$ is finite, $\bar{R}^{m}$ is the Banach's dual.

$\bar{R}^{m}$ is a Nakano space with its modular:

$$
\bar{m}(\bar{x})=\sup _{x \in R}\{\bar{x}(x)-m(x)\}
$$

for every $\bar{x} \in \bar{R}^{m}$.

\section{REFLEXIVITIES OF MODULARS AND THEIR NORMS}

In the sequel of this paper we assume that $R$ satisfies the following condition: If $\bar{x}[p]=0$ for every $\bar{x} \in \bar{R}$, then $[p]=0$.

2.1. Reflexivity of modulars. Mori-Amemiya-Nakano's theorem is as follows:

If a norm $\|x\|$ on $R$ which is compatible with order is universally semi-continuous $\left(0 \leqq x_{\lambda} \uparrow_{\lambda \in \Lambda} x\right.$ implies $\left.\|x\|=\sup _{\lambda \in \Lambda}\left\|x_{\lambda}\right\|\right)$, then

$$
\|x\|=\sup _{\mu(x) \leqq 1 ; \tilde{x} \in \bar{R}}|\bar{x}(x)|
$$

where $\mu(\bar{x})=\sup _{\|x\| \text { s1 }}|\bar{x}(x)|$.

Since the norms defined by modular are universally semi-continuous, they are reflexive. Our aim is to prove the Nakano's reflexivity theorem of modulars:

$$
m(x)=\sup _{\bar{x} \in \bar{R}^{m}}\{\bar{x}(x)-\bar{m}(\bar{x})\}
$$

by using the above Mori-Amemiya-Nakano's theorem. Namely, in this section we assume the following conditions:

$\mathrm{N} 1 .\|z\|=\sup _{\|x \mid\| \leq 1}|\bar{x}(x)|$ and $\left|\left\|\bar{x}\left|\|=\sup _{\|x\| \leq 1}\right| \bar{x}(x) \mid\right.\right.$ are the first and second norm of $\bar{R}^{m}$ respectively;

N2. $\|x\|=\sup _{\||\bar{x}|\| \leqq 1}|\bar{x}(x)|$ and $\left\||| x\left|\|=\sup _{\|\bar{x}\| \leq 1}\right| \bar{x}(x) \mid\right.$.

Putting 


$$
m_{\lambda}(x)=\frac{1}{\lambda} m(x)
$$

for every positive number $\lambda$, we obtain a system of modulars on $R$. Let $\|x\|_{\lambda}$ and $\|x \mid\|_{\lambda}$ be the first and second norms of $m_{\lambda}$ respectively. Namely,

$$
\|x\|_{\lambda}=\inf _{\xi>0} \frac{\lambda+m(\xi x)}{\xi} \text { and }\|\| x \|_{\lambda}=\inf _{m(\xi x) \leqq \lambda} \frac{1}{|\xi|} .
$$

Then, we have the following properties:

$$
\|x\|_{\lambda}=\inf _{\rho>0}(\lambda+\rho)\|x\|_{\rho} \quad \text { for every } \lambda>0 .
$$

In fact,

$(2)$

$$
\begin{aligned}
\|x\|_{\lambda} & =\inf _{\xi>0} \frac{\lambda+m(\xi x)}{\xi}=\inf _{\rho>0} \cdot \inf _{m(\xi x) \leqq \rho} \frac{\lambda+\rho}{|\xi|}=\inf _{\rho>0}(\lambda+\rho)\|x\|_{\rho} . \\
m(x) & =\sup _{\lambda>0}\left\{\|x\|_{\lambda}-\lambda\right\} .
\end{aligned}
$$

We need only prove the inequality:

$$
m(x) \leqq \sup _{\lambda>0}\left\{\|x\|_{\lambda}-\lambda\right\}
$$

because the inverse inequality follows immediately from the definition of $\|x\|_{\lambda}$. For any $\rho>0$ such that

$$
\rho>\|x\|_{\lambda}-\lambda
$$

we have

$$
\rho+\lambda>\|x\|_{\lambda}=\sup _{\||| \bar{x}\| \|_{\lambda} \leqq 1}|\bar{x}(x)| .
$$

Therefore, $|\bar{x}(x)| \leqq(\rho+\lambda)\left|\|\bar{x} \mid\|_{\lambda}\right.$ for every $\lambda>0$, from which and (1) follows that $|\bar{x}(x)| \leqq\|\bar{x}\|_{\rho}$. Hence it follows that \|\|$x \|_{\rho} \leqq 1$, namely, $m(x) \leqq \rho$. Therefore the required inequality is obtained.

$$
\|x\|\left\|_{\lambda}=\sup _{\rho>0}\right\| x \|_{\rho} /(\lambda+\rho) \quad \text { for every } \lambda>0 .
$$

For any $\xi>0$ such that

$$
\xi>\sup _{\rho>0}\|x\|_{\rho} /(\lambda+\rho),
$$

we have

$$
\lambda>\left\|\frac{x}{\xi}\right\|_{\rho}-\rho \quad \text { for every } \rho>0 .
$$


Therefore, by (2) we have $\lambda \geqq m(x / \xi)$, namely, $\xi \geqq\|x \mid\| \|_{\lambda}$, which shows the inequality

$$
\|x \mid\|\left\|_{\lambda} \leqq \sup _{\rho>0}\right\| x \|_{\rho} /(\lambda+\rho)
$$

is true. The inverse inequality follows from (1).

Proof of the reflexivity $(*)$. For any $\lambda>0$ such that

$$
\lambda>\sup _{\bar{x} \in \bar{R}^{m}}\{\bar{x}(x)-\bar{m}(\bar{x})\},
$$

we have

$$
|\bar{x}(x)| \leqq \lambda+\bar{m}(\bar{x}) .
$$

Therefore, by our assumption we have

$$
\|x\|_{\rho} \leqq \lambda+\rho \quad \text { for every } \rho>0,
$$

from which and (3) we have

$$
\|x\|_{\lambda} \leqq 1, \text { namely, } m(x) \leqq \lambda .
$$

Hence the following inequality is true;

$$
m(x) \leqq \sup _{\bar{x} \in \bar{R}^{m}}\{\bar{x}(x)-\bar{m}(\bar{x})\} .
$$

The inverse inequality is an immediate consequence of the definition of $\bar{m}(\bar{x})$.

REMark. The norms $\|x \mid\|_{\lambda}$ defined above satisfy the following three conditions:

(1) ||$|x+y| \|(\lambda+\rho) / 2 \leqq \operatorname{Max}\left\{\left|\left\|x\left|\left\|_{\lambda},\right\|\right||y|\right\|_{\rho}\right\}\right.$;

(2) If $\|x\| \|_{\lambda}=0$ for every $\lambda>0$, then $x=0$;

(3) If $x \cap y=0$, then $\|\mid x+y\| \|_{\lambda+\rho} \leqq$ Max. $\left\{\left\||| x\left|\|\|_{\lambda},\|\mid y\|_{\rho}\right\}\right.\right.$, and for any $\xi>0$ there exist $\lambda, \rho>0$ such that

$$
\||| x+y \mid\| \xi \geqq \operatorname{Max} .\left\{\left\|x\left|\left\|_{\lambda},\right\|\right| \mid y\right\|_{\rho}\right\} .
$$

Conversely, if on a universally continuous semi-ordered linear space there exists a system of semi-norms which satisfies the above three conditions, we can define a modular on the space.

2.2. Reflexivity of norms. The purpose of this section is to prove $N i$ $(i=1,2)$ of the preceding section by $\left({ }^{*}\right)$. But, as may be seen by the proofs, $(*)$ is necessary only for $\mathrm{N} 2$.

$$
\|\bar{x}\|=\sup _{m(x) \leqq 1}|\bar{x}(x)|=\inf _{\xi>0} \frac{1+\bar{m}(\xi \bar{x})}{\xi} .
$$


To prove this, we make use of Theorem 6.4 of Nakano [3], which is, of course, true in the case of $\bar{R}^{m}$. Put

$$
\alpha=\|\bar{x}\|=\inf _{\xi>0} \frac{1+\bar{m}(\xi \bar{x})}{\xi},
$$

then, since $\alpha \xi \leqq 1+\bar{m}(\xi \bar{x})$ for any $\xi>0$, for any $\epsilon>0$ there exists $x_{0} \in R$ such that

$$
\bar{x}\left(x_{0}\right)=\alpha \text { and } m\left((1-\epsilon) x_{0}\right) \leqq 1 .
$$

Therefore, we have

$$
\|\bar{x}\| \leqq \sup _{m(x) \leqq 1}|\bar{x}(x)|
$$

On the other hand, since

$$
|\xi \bar{x}(x)| \leqq m(x)+\bar{m}(\xi \bar{x}) \quad \text { for every } \xi>0,
$$

we have

$$
\sup _{m(x) \leqq 1}|\bar{x}(x)| \leqq \frac{1}{\xi}\{1+\bar{m}(\xi \bar{x})\} \quad \text { for every } \xi>0 .
$$

Hence it follows that

$$
\begin{gathered}
\sup _{m(x) \leqq 1}|\bar{x}(x)| \leqq\|\bar{x}\| . \\
\||\bar{x}|\|=\sup _{\|x\| \leqq 1}|\bar{x}(x)|=\inf _{\bar{m}(\xi \bar{x}) \leqq 1} \frac{1}{|\xi|} .
\end{gathered}
$$

For any $\alpha>0$ such that

$$
\alpha>\sup _{\|x\| \leqq 1}|\bar{x}(x)|,
$$

we have

$$
\left|\frac{1}{\alpha} \bar{x}(x)\right| \leqq\|x\| \leqq 1+m(x)
$$

from which we have

$$
\bar{m}\left(\frac{1}{\alpha} \bar{x}\right)=\sup _{x \in R}\left\{\frac{1}{\alpha} \bar{x}(x)-m(x)\right\} \leqq 1,
$$

namely, || $\bar{x}|| \mid \leqq \alpha$. Therefore we have

$$
|||\bar{x}| \mid\rfloor \sup _{|| x|| \leqq 1}|\bar{x}(x)| .
$$

On the other hand, if we put $\alpha=\||\bar{x}|\|$, we have 


$$
\bar{m}\left(\frac{1}{\alpha} \bar{x}\right) \leqq 1
$$

and hence it follows that

$$
\left|\frac{1}{\alpha} \bar{x}(x)\right| \leqq 1+m(x) \quad \text { for every } x \in R .
$$

Therefore we have

$$
\left|\frac{1}{\alpha} \bar{x}(x)\right| \leqq\|x\|
$$

which shows that

$$
\sup _{\|x\| \leqq 1}|\bar{x}(x)| \leqq \alpha .
$$

Thus we could prove the equality

$$
\left\|\left|x \|=\sup _{\|\bar{x}\| \leqq 1}\right| \bar{x}(x) \mid \text { and }\right\| x \|=\sup _{\|x \bar{x}\| \leqq 1}|\bar{x}(x)| .
$$

We can consider every element $x$ as an element of $x^{\bar{R}} \in\left[\bar{R}^{m}\right]^{m}$, namely,

$$
\bar{x}(x)=x^{R}(\bar{x}) \quad \text { for every } \bar{x} \in \bar{R}^{m} .
$$

The equality $\left(^{*}\right)$ shows that $m(x)=[\bar{m}]-\left(x^{\bar{R}}\right)$. Since $(\xi x)^{\bar{R}}=\xi x^{\bar{R}}$, we have

$$
\|x\|=\inf _{\xi>0} \frac{1+m(\xi x)}{\xi}=\inf _{\xi>0} \frac{1+[\bar{m}]-\left(\xi x^{\bar{R}}\right)}{\xi}=\left\|x^{\bar{R}}\right\|,
$$

and

$$
\left\||| x||=\inf _{m(\xi x) \leqq 1} \frac{1}{|\xi|}=\inf _{[m]-(\xi x \bar{R}) \leqq 1} \frac{1}{|\xi|}=\right\||| x^{\bar{R}} \mid \| .
$$

Therefore, by (1) and (2), we have

$$
\|\| x \|=\sup _{\|\bar{x}\| \leqq 1}|\bar{x}(x)| \text { and } \quad\|x\|=\sup _{\|\mid \tilde{x}\| \| \leqq 1}|\bar{x}(x)| .
$$

3. NORMS OF LINEAR FUNCTIONALS

3.1. Mazur's functional. In the theory of Banach spaces, Mazur [1] has proved that, if $\|x\|=\|y\|=1$, then the limit:

$$
\phi_{x}(y)=\lim _{\eta \rightarrow 0+} \frac{1}{\eta}(\|x+\eta y\|-\|x\|)
$$

exists and the functional $\phi_{x}$ is bounded and linear. The purpose of this section is to obtain an integral representation of the Mazur's functional $\phi_{x}$. In this 
section, we must assume that the modular spectrum $\omega(\xi, a, \mathfrak{p})$ is a differentiable function of $\xi$.

Let $m$ be finite and $a$ be a simple element. Then we have

$$
f(\eta)=m\left(\frac{a+\eta[a] x}{\|\| a+\eta[a] x \|}\right)=1
$$

for any element $x \geqq 0$. On the other hand,

$$
f(\eta)=\int_{[a]} \omega(g(\eta, \mathfrak{p}), a, \mathfrak{p}) m(d \mathfrak{p} a),
$$

where

$$
\begin{aligned}
g(\eta, \mathfrak{p}) & =1+\eta\left(\frac{x}{a}, \mathfrak{p}\right) / h(\eta), \\
h(\eta) & =\|\| a+\eta[a] x \| \mid .
\end{aligned}
$$

If we denote the right-hand derivative by $D^{+}$, we have

$$
D^{+} \omega(g(\eta, \mathfrak{p}), a, \mathfrak{p})=\pi(g(\eta, \mathfrak{p}), a, \mathfrak{p}) D^{+} g(\eta, \mathfrak{p}),
$$

where

$$
\pi(\xi, a, \mathfrak{p})=\inf _{\epsilon>0} \frac{1}{\epsilon}\{\omega(\xi+\epsilon, a, \mathfrak{p})-\omega(\xi, a, \mathfrak{p})\}
$$

and

$$
D^{+} g(\eta, \mathfrak{p})=\frac{1}{h(\eta)^{2}}\left\{h(\eta)\left(\frac{x}{a}, \mathfrak{p}\right)-D^{+} h(\eta)\left(1+\eta\left(\frac{x}{a}, \mathfrak{p}\right)\right)\right\} .
$$

Therefore,

$$
\begin{aligned}
0= & D^{+} f(\eta) \\
= & \frac{1}{h(\eta)}\left\{\int_{[a]} \pi(g(\eta, \mathfrak{p}), a, \mathfrak{p})\left(\frac{x}{a}, \mathfrak{p}\right) m(d \mathfrak{p} a)\right. \\
& \left.-D^{+} h(\eta) \int_{[a]} \pi(g(\eta, \mathfrak{p}), a, \mathfrak{p}) g(\eta, \mathfrak{p}) m(d \mathfrak{p} a)\right\} .
\end{aligned}
$$

Let us assume $\||a|\|=1$. Then, since $h(\eta) \geqq 1$, we have

$$
\left[D^{+} h(\eta)\right]_{\eta=0}=\frac{1}{\pi(1 / a)} \int_{[a]} \pi(1, a, \mathfrak{p})\left(\frac{x}{a}, \mathfrak{p}\right) m(d \mathfrak{p} a),
$$

where

$$
\pi(\xi / a)=\inf _{\epsilon \leqq 0} \frac{1}{\epsilon}\{m((\xi+\epsilon) a)-m(\xi a)\}
$$


Thus we can state the following theorem:

THEOREM 3.1. If $m$ is finite and the modular spectrum is differentiable, then, for a simple element a such that $\||||| \mid=1$, the Mazur's functional of the second norm can be expressed by the following formula:

$$
\phi_{a}(x)=\frac{1}{\pi(1 / a)} \int_{[a]} \pi(1, a, \mathfrak{p})\left(\frac{x}{a}, \mathfrak{p}\right) m(d \mathfrak{p} a),
$$

and hence $\phi_{a} \in \bar{R}^{m}$ and $\bar{m}\left(\pi(1 / a) \phi_{a}\right)=\pi(1 / a)-1$.

REMARK. For the first norm, we can not apply the same method, because $m(x /\|x\|)$ is not necessarily constant. For example, if we take the sequence space $l(1,2, \cdots)$ of all sequences $\left(\xi_{\nu}\right)$ with

$$
\sum_{\nu=1}^{\infty}\left|\alpha \xi_{\nu}\right|^{\nu}<+\infty
$$

for some $\alpha>0$, then for the elements:

$$
e_{1}=(1,0, \cdots) ; \quad e_{2}=(0,1,0, \cdots) ; \quad \cdots
$$

we have

$$
m\left(\frac{e_{\nu}}{\left\|e_{\nu}\right\|}\right)=\frac{1}{\nu}\left(\frac{\nu}{\nu-1}\right)^{\nu-1} .
$$

But, if $m(x /\|x\|)$ is constant for every $x \in R$, such as $L_{p}$-spaces, then it is easily seen that the Mazur's functional of the first norm coincides with that of the second norm.

3.2. Norms of a linear functional of integral type. The linear functional appeared in the preceding section:

$$
a^{\bar{R}}(x)=\int_{[a]} \pi(1, a, \mathfrak{p})\left(\frac{x}{a}, \mathfrak{p}\right) m(d \mathfrak{p} a)
$$

plays very important roles in the theory of Nakano spaces. For example, by the Theorem 39.1 of Nakano [1], if a is simple and $m((1+\epsilon) a)<+\infty$ for some $\epsilon>0$, we have

$$
a^{\bar{R}}(a)=m(a)+\bar{m}\left(a^{\bar{R}}\right),
$$

namely, by the functional $a^{\bar{R}}$, the Young's inequality takes equality sign.

In the case of $L_{p}$-spaces $(p>1)$, putting its modular as

$$
m(x)=\frac{1}{p} \int_{0}^{1}|x(t)|^{p} d t,
$$

since 


$$
a^{\bar{R}}(x)=\int_{0}^{1} x(t)|a(t)|^{p-1} \operatorname{sign} a(t) d t
$$

we have

$$
\left\|a^{\bar{R}}\right\| \cdot\left|\left\|a \left|\left\|=\left|\left\|a^{\bar{R}}|\|\cdot\| a \|=| a^{\bar{R}}(a) \mid .\right.\right.\right.\right.\right.\right.
$$

But in general cases, we can not have such good relations. For example, take a Nakano sequence space $l\left(f_{1}, f_{2}, \cdots\right)$ determined by the following functions:

$$
f_{1}(\xi)=\xi ; \quad f_{2}(\xi)=\frac{1}{2} \xi^{2} ;
$$

and arbitrary $f_{\nu}(\nu \geqq 3)$. Then, for

$$
a=(1,1,0, \cdots)
$$

we have $a^{\bar{R}}(a)=2$ and

$$
\begin{array}{rlrl}
\|a\| & =1+2^{1 / 2} ; & \|a\| \| & =\left(3^{1 / 2}-1\right)^{-1} ; \\
\left\|a^{\bar{R}}\right\| & =1.5 ; \quad\left\|\mid a^{\bar{R}}\right\| & =1 .
\end{array}
$$

Therefore, $\left\|a^{\bar{R}}\right\| \cdot\left\||| a|\|\neq\|| a^{\bar{R}} \mid\right\| \cdot\|a\|$ and $a^{\bar{R}}(a)<\operatorname{Min}\left\{\left\|a^{\bar{R}}\right\| \cdot\|\mid\| a\|\|,\left\|a^{\bar{R}}\right\|\|\cdot\| a \|\right\}$.

We will study in what cases we can have the equality relations. We begin by a lemma which is an improvement of Theorem 6.4 of Yamamuro [2].

Lemma 3.2.1. Let $m$ be finite and increasing:

$$
\lim _{\xi \rightarrow \infty} \frac{m(\xi a)}{\xi}=+\infty
$$

then there exists a number $\alpha>0$ such that

$$
\|\alpha a\|=1+m(\alpha a) .
$$

Proof. By the definition of the first norm $\|a\|$, we can find a sequence $\xi_{\nu}(\nu=1,2, \cdots)$ such that

$$
\left(1+\frac{1}{\nu}\right)\|a\|>1+m\left(\xi_{\nu} a\right) / \xi_{\nu} .
$$

At first we will show that $\xi_{\nu}^{-1}(\nu=1,2, \cdots)$ are bounded. In fact, if $\inf _{\nu \geq 1} \xi_{\nu}=0$, then for a sequence $\xi_{\nu_{\mu}}(\mu=1,2, \cdots)$ such that $\lim _{\mu \rightarrow \infty} \xi_{\nu_{\mu}}=0$, we have

$$
\frac{1}{\xi_{\nu_{\mu}}} \leqq \frac{1+m\left(\xi_{\nu_{\mu}} a\right)}{\xi_{\nu_{\mu}}}<\left(1+\frac{1}{\nu_{\mu}}\right)\|a\| \leqq 2\|a\|,
$$

which is a contradiction. Next, let $\sup _{\nu \geq 1} \xi_{\nu}=+\infty$. Then, for a subsequence $\xi_{\nu_{\mu}}(\mu=1,2, \cdots)$ such that $\lim _{\mu \rightarrow \infty} \xi_{\nu_{\mu}}=+\infty$, we have 


$$
\frac{m\left(\xi_{\nu_{\mu}} a\right)}{\xi_{\nu_{\mu}}} \leqq \frac{1+m\left(\xi_{\nu_{\mu}} a\right)}{\xi_{\nu_{\mu}}} \leqq 2\|a\|,
$$

which is also a contradiction, since

$$
\lim _{\mu \rightarrow \infty} \frac{m\left(\xi_{\nu_{\mu}} a\right)}{\xi_{\nu_{\mu}}}=+\infty .
$$

Therefore, there exist $\gamma_{1}$ and $\gamma_{2}$ such that

$$
0<\gamma_{1}<\xi_{\nu}<\gamma_{2},
$$

and hence, we can find a sequence $\xi_{\nu_{\mu}}(\mu=1,2, \cdots)$ and $\xi_{0}$ such that

$$
\lim _{\mu \rightarrow \infty} \xi_{\nu_{\mu}}=\xi_{0} \text { and } \gamma_{1} \leqq \xi_{0} \leqq \gamma_{2}
$$

Since $m(\xi a)$ is continuous with respect to $\xi>0$, we have

$$
\lim _{\mu \rightarrow \infty} \frac{1+m\left(\xi_{\nu_{\mu}} a\right)}{\xi_{\nu_{\mu}}}=\frac{1+m\left(\xi_{0} a\right)}{\xi_{0}} \leqq\|a\| .
$$

As the converse inequality is a direct consequence of the definition of $\|a\|$, we have

$$
1+m\left(\xi_{0} a\right)=\left\|\xi_{0} a\right\| .
$$

REMARK. In general, such number $\alpha$ does not always exist. In fact, in the spaces $L$ and $M$, we can define modulars for which $m(x)=\|x\|=\|x\| \mid$ for every $x \in R$. (See Nakano [1, p. 184], and Yamamuro [1]).

Theorem 3.2.1. If $m(a)=1$ and $1+\bar{m}(\bar{a})=\bar{a}(a)$, then $\bar{a}(a)=\|\bar{a}\|$. Conversely, when $\bar{m}$ is finite and increasing, then

$$
1+\bar{m}(\alpha \bar{a})=\alpha \bar{a}(a) \quad \text { for some } \alpha>0,
$$

if $\|\bar{a}\|=\bar{a}(a)$ and $m(a)=1$.

Proof. If $m(a)=1$ and $\bar{m}(\bar{a})+1=\bar{a}(a)$, then by the definition of the first norm, we have

$$
\|\bar{a}\| \leqq 1+\bar{m}(\bar{a})=\bar{a}(a) \leqq\|\bar{a}\|,
$$

namely, $\|\bar{a}\|=\bar{a}(a)$. Conversely, if $\bar{m}$ is finite and increasing, then by Lemma 3.2.1, we have

$$
\alpha\|\bar{a}\|=1+\bar{m}(\alpha \bar{a})
$$
for some $\alpha>0$,

hence it follows that $\alpha \bar{a}(a)=1+\bar{m}(\alpha \bar{a})$.

THEOREM 3.2.2. If $m(a)=1$, then

$$
\left\|a^{\bar{R}}\right\|=a^{\bar{R}}(a)=\pi(1 / a) .
$$


Proof. Since $1+\bar{m}\left(a^{\bar{R}}\right)=a^{\bar{R}}(a)$, by the preceding theorem, we have $\left\|a^{\bar{R}}\right\|$ $=a^{\bar{R}}(a)$. On the other hand,

$$
a^{\bar{R}}(a)=\int_{[a]} \pi(1, a, \mathfrak{p}) m(d \mathfrak{p} a)=\pi(1 / a),
$$

by Theorem 2.1 of Yamamuro [2].

ThEOREM 3.2.3. Let the first norm of the conjugate space be strictly convex. Then

$$
\left\|a^{\bar{R}}\right\| \cdot\|a\| \|=a^{\bar{R}}(a)
$$

if and only if

$$
\pi\left(1 /[p] \frac{a}{\||\|a\||}\right) / \pi(1 /[p] a)=\text { constant for every }[p] \leqq[a] .
$$

Proof. Put $\phi_{a}=(a / \||| a|| \mid)^{\bar{R}}$. Then

$$
\phi_{a}(x)=\int_{[a]} \pi\left(\frac{1}{\||a|\|}, a, \mathfrak{p}\right)\left(\frac{x}{a}, \mathfrak{p}\right) m(d \mathfrak{p} a),
$$

and $1+\bar{m}\left(\phi_{a}\right)=\phi_{a}(a /\|a\| \|)$. Therefore by Theorem 3.2.1, we have

$$
\phi_{a}(a)=\left\|\phi_{\alpha}\right\| \cdot\||| a\| \mid .
$$

If $a^{\bar{R}}(a)=\left\|a^{\bar{R}}\right\| \cdot\|\mid\| a\|\|$, then, since the first norm of the conjugate space is strictly convex, we have $\alpha a^{\bar{R}}=\phi_{a}$ for some $\alpha>0$. Therefore,

$$
\begin{aligned}
\pi(1 /[p] a /\|a\| \|) & =\frac{1}{\|\| a \|} \int_{[p]} \pi\left(\frac{1}{\|\mid\| a \|}, a, p\right) m(d p a) \\
& =\phi_{a}\left(\frac{[p] a}{\|\| a\|\|}\right)=\alpha a^{\bar{R}}\left(\frac{[p] a}{\|\| a\|\|}\right)=\frac{\alpha}{\|\| a\|\|} \pi(1 /[p] a)
\end{aligned}
$$

for every $[p] \leqq[a]$. Conversely, for the number $\alpha$ such that

$$
\alpha=\pi\left(\frac{1}{\|\bar{a}\| \|} /[p] a\right) / \pi(1 /[p] a),
$$

we have

$$
\begin{aligned}
\phi_{a}(x) & =\int_{[a]} \pi\left(\frac{1}{\|a\|}, a, \mathfrak{p}\right)\left(\frac{x}{a}, \mathfrak{p}\right) m(d \mathfrak{p} a) \\
& =\alpha \int_{[a]} \pi(1, a, \mathfrak{p})\left(\frac{x}{a}, \mathfrak{p}\right) m(d \mathfrak{p} a) \\
& =\alpha a^{\bar{R}}(x)
\end{aligned}
$$


for every $x \in R$, namely, $\phi_{a}=\alpha a^{\bar{R}}$. Therefore, $\left\|a^{\bar{R}}\right\| \cdot\|\mid\| a \|=a^{\bar{R}}(a)$.

REMARK. If $a$ is a constant element (see Nakano $[1, \S 55]$ ), then $\pi((1 /\|a\| \|) /[p] a) / \pi(1 /[p] a)$ is constant for every $[p] \leqq[a]$. In the spaces $L_{p}(p \geqq 1)$, every element is constant. In the Orlicz spaces $L_{\Phi}$, for any elements $x, y \in L_{\Phi}$, there exists a constant element $a \in L_{\Phi}$ such that $[a] x \neq 0,[a] y \neq 0$.

Next we will calculate the second norm of $a^{\bar{R}}$.

LemmA 3.2.2. If $\pi(1 / a)=1+m(a)$, then $\left\|a^{\bar{R}}\right\| \|=1$.

Proof. Since

$$
\|a\| \leqq 1+m(a)=\pi(1 / a)=a^{\bar{R}}(a),
$$

we have $\left\|\left|a^{\bar{R}}\right|\right\| \geqq 1$. On the other hand,

$$
\bar{m}\left(a^{\bar{R}}\right)=a^{\bar{R}}(a)-m(a)=1, \text { namely, } \quad\left\|a^{\bar{R}}\right\| \leqq 1 .
$$

Lemma 3.2.3. If $\pi(1 / \alpha a)=1+m(\alpha a)$ for some $\alpha>0$, then $\|a\|=\pi(\alpha / a)$.

Proof. Since

$$
\|\alpha a\| \leqq 1+m(\alpha a)=\pi(1 / \alpha a),
$$

we have $\|a\| \leqq \pi(\alpha / a)$. On the other hand, since

$$
(\alpha a)^{\bar{R}}(\alpha a)=\pi(1 / \alpha a)=m(\alpha a)+\bar{m}\left((\alpha a)^{\bar{R}}\right),
$$

we have $\bar{m}\left((\alpha a)^{\bar{R}}\right)=1$. Therefore,

$$
\|\alpha a\| \geqq(\alpha a)^{\bar{R}}(\alpha a)=\pi(1 / \alpha a) .
$$

THEOREM 3.2.4. Let the second norm of the conjugate space be strictly convex and

$$
\pi(1 / \alpha a)=1+m(\alpha a)
$$

for some $\alpha>0$. Then

$$
\left\|a^{\bar{R}}\right\| \mid \cdot\|a\|=a^{\bar{R}}(a)
$$

if and only if

$$
\pi(\alpha /[p] a) / \pi(1 /[p] a)=\text { constant for every }[p] \leqq[a] .
$$

Proof. Let us assume that $\left\|a^{\bar{R}} \mid\right\| \cdot\|a\|=a^{\bar{R}}(a)$. Since $(\alpha a)^{\bar{R}}(a)=\pi(\alpha \mid a)$ $=\|a\|$ by Lemma 3.2.3, we have

$$
\left((\alpha a)^{\bar{R}}+a^{\bar{R}}\right)(a)=\|a\|+\|a\| \cdot\left\||| a^{\bar{R}}\right\|\|=\| a \|\left(\| \| ( \alpha a ) ^ { \overline { R } } \left\|\left|+\left\|\mid a^{\bar{R}}\right\| \|\right),\right.\right.
$$

because, by Lemma 3.2.2, we have $\left|\left\|(\alpha a)^{\bar{R}} \mid\right\|=1\right.$. On the other hand, we have

$$
\left((\alpha a)^{\bar{R}}+a^{\bar{R}}\right)(a) \leqq\|a\| \cdot\|\|(\alpha a)^{\bar{R}}+a^{\bar{R}} \| .
$$

Therefore, 


$$
\left\|(\alpha a)^{\bar{R}}+a^{\bar{R}}\left|\|=\|\left\|(\alpha a)^{\bar{R}}\right\|\right|+\right\| a^{\bar{R}} \| \mid,
$$

hence it follows that

$$
(\alpha a)^{\bar{R}}=\beta a^{\bar{R}} \quad \text { for some } \beta>0,
$$

because the second norm of the conjugate space $\bar{R}^{m}$ is strictly convex. Therefore,

$$
\pi(\alpha /[p] a)=(\alpha a)^{\bar{R}}([p] a)=\beta a^{\bar{R}}([p] a)=\beta \pi(1 /[p] a)
$$

for every $[p] \leqq[a]$.

Next, let us assume

$$
\pi(\alpha /[p] a)=\beta \pi(1 /[p] a)
$$

for some $\beta>0$. Then, since $(\alpha a)^{\bar{R}}=\beta a^{\bar{R}}$, we have

$$
\beta\left|\left\|a ^ { \overline { R } } \left|\left\|=\left|\left\|(\alpha a)^{\bar{R}} \mid\right\|=1, \text { namely, } \quad\left\|a^{\bar{R}}\right\|\right|=1 / \beta,\right.\right.\right.\right.
$$

and

$$
\beta=\pi(\alpha / a) / \pi(1 / a)=\|a\| / a^{\bar{R}}(a) .
$$

Therefore we obtain $a^{\bar{R}}(a)=\left\|\left|a^{\bar{R}}\right|\right\| \cdot\|a\|$.

REMARK 1. Even if $m$ is strictly convex (see Appendix II), the number $\alpha>0$ such that $\alpha \pi(\alpha / a)=1+m(\alpha a)$ does not always exist. For example, take a convex function $f(\xi)$ such that

$$
f(\xi)=\left\{\begin{array}{l}
\xi^{2} / 2 \text { if } 0 \leqq \xi \leqq 1 \\
\xi^{2}-1 / 2 \text { if } \quad \xi \geqq 1
\end{array}\right.
$$

This is a strictly convex modular on real line and, for any $\xi$, the equality

$$
\xi f^{\prime}(\xi)=1+f(\xi)
$$

does not hold.

REMARK 2. Let $\tau(\xi \mid a)$ be the left-hand derivative of $m(\xi a)$. Then, if $\|\alpha a\|=1+m(\alpha a)$, we have

$$
\tau(\alpha / a) \leqq \frac{1+m(\alpha a)}{\alpha} \leqq \pi(\alpha / a) .
$$

Therefore, if $m(\xi a)$ is differentiable at $\alpha$, then

$$
\pi(1 / \alpha a)=1+m(\alpha a) .
$$

REMARK 3. If $m$ is strictly convex, then

$$
\|\alpha a\|=\pi(\alpha / a)
$$

for and only for $\alpha$ such that $\pi(\alpha / a)=1+m(\alpha a)$.

Finally, we will introduce a different condition:

Theorem 3.2.5. If $m(a)=\sup _{\|x\|=1} m(x)$ and $\|a\| \leqq 1$, then $\left\|a^{\bar{R}}\right\|=a^{\bar{R}}(a)$. 
Proof. Since

$$
\bar{m}\left(a^{\bar{R}}\right)=a^{\bar{R}}(a)-m(a) \geqq a^{\bar{R}}(x)-m(x)
$$

for every $x \in R$, we have

$$
\sup _{\|x\|=1}\left|a^{\bar{R}}(x)\right| \leqq a^{\bar{R}}(a)-m(a)+\sup _{\|x\|=1} m(x)=a^{\bar{R}}(a) .
$$

Therefore, $\left\|\left|a^{\bar{R}}\right|\right\|=a^{\bar{R}}(a)$, since $\|a\|=1$.

REMARK 1. Therefore, if $m(x /\|x\|)=$ constant for every $x \in R$, which is true in the case of $L_{p}(p \geqq 1)$, we have $\left\|a^{\bar{R}}\right\|=a^{\bar{R}}(a)$, if $\|a\|=1$.

REMARK 2. $\sup _{\|x\|=1} m(x)=\sup _{\bar{x} \in \bar{R}^{m}}\{\|\| \bar{x} \|-\bar{m}(\bar{x})\}$.

In fact, using the norm system of $\$ 2$, we can prove that,

$$
\rho_{0}=\sup _{\left\||| x\left|\left\|_{\rho} \leqq\right\| x \|\right|\right.} \rho=\inf _{\||\bar{x}|\| \leqq\|\tilde{x} \mid\|_{\rho}} \rho
$$

where $\rho_{0}=\sup _{\|x\|=1} m(x)$, and $\left\|\left|\bar{x}\|\mid \leqq\| \bar{x} \|_{\rho}\left(\bar{x} \in \bar{R}^{m}\right)\right.\right.$ if and only if

$$
\| \bar{x}||-\bar{m}(\bar{x}) \leqq \rho \quad \text { for every } \bar{x} \in \bar{R}^{m} .
$$

\section{APPENDIX I. InTEgRAL REPRESENTATION OF LINEAR FUNCTIONALS}

In the sequel, let $R$ be a universally continuous semi-ordered linear space and a function $\mu([p])$ of projectors $[p]$, be a countably additive measure, namely,

(1) $0<\mu([p])<+\infty$ for $[p] \neq 0$;

(2) $\mu([p]+[q])=\mu([p])+\mu([q])$;

(3) If $\left[p_{\nu}\right] \downarrow_{\nu=1}^{\infty} 0$, then $\lim _{\nu \rightarrow \infty} \mu\left(\left[p_{\nu}\right]\right)=0$.

Theorem A.1. For $\bar{x} \in \bar{R}$ and $a \in R$, there exists an almost finite, continuous function $\bar{x}_{a}(\mathfrak{p})$ on $U_{[a]}$, such that

$$
\bar{x}([p] x)=\int_{[p]}\left(\frac{x}{a}, \mathfrak{p}\right) \bar{x}_{a}(\mathfrak{p}) d \mu
$$

for every $x \in R$ such that $[x] \leqq[a]$.

Proof. Let $[x] \leqq[a]$. By a similar argument employed in the proof of Theorem 36.1 of Nakano [1], we can prove that the limit:

$$
\bar{x}_{a}(\mathfrak{p})=\lim _{[p] \rightarrow \mathfrak{p}} \frac{\bar{x}([p] a)}{\mu([p])}
$$

exists for every $\mathfrak{p} \in U_{[a]}$ and $\bar{x}_{a}(\mathfrak{p})$ is almost finite, continuous in the extended sense in $U_{[a]}$. On the other hand, by Theorem 54.3 of Nakano [2], we have

$$
\lim _{[p] \rightarrow \mathfrak{p}} \frac{\bar{x}([p] x)}{\bar{x}([p] a)}=\left(\frac{x}{a}, \mathfrak{p}\right) \quad \text { if } \mathfrak{p} \in U_{[a]} C_{\bar{x}} .
$$

Therefore, 


$$
\lim _{[p] \rightarrow \mathfrak{p}} \frac{\bar{x}([p] x)}{\mu([p])}=\left(\frac{x}{a}, \mathfrak{p}\right) \bar{x}_{a}(\mathfrak{p}),
$$

from which it follows that

$$
\bar{x}([p] x)=\int_{[p]}\left(\frac{x}{a}, \mathfrak{p}\right) \bar{x}_{a}(\mathfrak{p}) d \mu .
$$

In the case of Nakano spaces, we can have more precise information about $\bar{x}_{a}(\mathfrak{p})$ :

Theorem A.2. Let $R$ be a Nakano space and a be a simple element such that $m((1+\epsilon) a)<+\infty$ for some $\epsilon>0$. Then, every $\bar{x} \in \bar{R}^{m}$ can be expressed as

$$
\bar{x}([p] x)=\int_{[p]} \pi(1, a, \mathfrak{p}) \omega(a, \mathfrak{p})\left(\frac{x}{a}, \mathfrak{p}\right)\left(\frac{\bar{x}}{a^{\bar{R}}}, \mathfrak{p}\right) d \mu
$$

for every $x$ such that $[x] \leqq[a]$. Here,

$$
\omega(a, \mathfrak{p})=\lim _{[p] \rightarrow \mathfrak{p}} \frac{m([p] a)}{\mu([p])},
$$

and

$$
a^{\overline{\boldsymbol{\pi}}}(x)=\int_{[a]} \pi(1, a, \mathfrak{p})\left(\frac{x}{a}, \mathfrak{p}\right) m(d \mathfrak{p} a) .
$$

Especially, in the case of $\mu([p])=m([p] a)$ for every $[p]$,

$$
\bar{x}([p] x)=\int_{[p]} \pi(1, a, \mathfrak{p})\left(\frac{x}{a}, \mathfrak{p}\right)\left(\frac{\bar{x}}{a^{\bar{R}}}, \mathfrak{p}\right) m(d \mathfrak{p} a) .
$$

Proof.

$$
\begin{aligned}
\bar{x}_{a}(\mathfrak{p})= & \lim _{[p] \rightarrow \mathfrak{p}} \frac{\bar{x}([p] a)}{\mu([p])}=\lim _{[p] \rightarrow \mathfrak{p}}\left\{\frac{\bar{x}([p] a)}{a^{\bar{R}}([p] a)} \cdot \frac{a^{\bar{R}}([p] a)}{\mu([p])}\right\} \\
& =\left(\frac{\bar{x}}{a^{\bar{R}}}, \mathfrak{p}\right) \cdot \pi(1, a, \mathfrak{p}) \cdot \omega(a, \mathfrak{p}) \cdot\left(\frac{x}{a}, \mathfrak{p}\right) .
\end{aligned}
$$

Appendix II. Strictly CONvex modulars ANd NORMS

Let $R$ be a Nakano space. A modular $m$ on $R$ is said to be strictly convex, if

$$
m\left(\frac{\xi+\eta}{2} x\right)=\frac{1}{2}\{m(\xi x)+m(\eta x)\} \quad \text { for } \xi, \eta>0
$$

implies $\xi=\eta$. If $m$ is strictly convex, then for any $x \neq y$, we have

$$
m(\alpha x+\beta y)<\alpha m(x)+\beta m(y), \quad \alpha+\beta=1, \alpha, \beta>0 .
$$


(See Nakano $[1, \S 61]$.)

If $m$ is strictly convex and

$$
\bar{m}(\bar{x})+m(x)=\bar{x}(x), \quad \bar{m}(\bar{x})+m(y)=\bar{x}(y),
$$

then $x=y$. Because, since

$$
\begin{aligned}
\bar{m}(\bar{x})+\frac{1}{2}\{m(x)+m(y)\} & =\bar{x}\left(\frac{x+y}{2}\right), \\
\bar{x}\left(\frac{x+y}{2}\right) & \leqq \bar{m}(\bar{x})+m\left(\frac{x+y}{2}\right),
\end{aligned}
$$

we have

$$
\frac{1}{2}\{m(x)+m(y)\}=m\left(\frac{x+v}{2}\right), \text { namely, } x=y .
$$

TheOREM A.3. If $m$ is strictly convex, then the second norm is strictly convex. If, moreover, $m$ is finite and increasing, then the first norm is strictly convex.

Proof. Suppose $\||x+y|\|=\|\| x|\|+\| y \|| \mid$ for some $x \in R$ and $y \in R$. Putting

$$
\alpha=\|x+y\|\left\|, \quad \alpha \alpha_{1}=\right\| x\|\|, \quad \alpha \alpha_{2}=\|\mid\| y \|,
$$

we have, since $m$ is finite,

$$
m\left(\alpha_{1} \frac{x}{\|x\|}+\alpha_{2} \frac{y}{\|y\|}\right)=m\left(\frac{x+y}{\|x+y\|}\right)=1,
$$

and

$$
\alpha_{1} m\left(\frac{x}{\|\mid x\| \|}\right)+\alpha_{2} m\left(\frac{y}{\|y\|}\right)=\alpha_{1}+\alpha_{2}=1
$$

Therefore, $\alpha_{2} x=\alpha_{1} y$, namely, the second norm is strictly convex.

Suppose $\|x+y\|=\|x\|+\|y\|$ for some $x \in R$ and $y \in R$. Then, by Lemma 3.2.1, there exist $\alpha>0$ and $\beta>0$ such that

$$
\|\alpha x\|=1+m(\alpha x) \text { and }\|\beta y\|=1+m(\beta y) .
$$

Therefore,

$$
\begin{aligned}
\|x+y\| & =\frac{1}{\alpha}+\frac{1}{\beta}+\frac{m(\alpha x)}{\alpha}+\frac{m(\beta y)}{\beta} \\
& =\frac{\alpha+\beta}{\alpha \beta}\left(1+\frac{\beta}{\alpha+\beta} m(\alpha x)+\frac{\alpha}{\alpha+\beta} m(\beta y)\right) .
\end{aligned}
$$

On the other hand, by the definition of the first norm, we have 


$$
\left\|\frac{\alpha \beta}{\alpha+\beta}(x+y)\right\| \leqq 1+m\left(\frac{\alpha \beta}{\alpha+\beta}(x+y)\right) .
$$

Therefore we obtain

$$
\left\|\frac{\alpha \beta}{\alpha+\beta}(x+y)\right\|=1+m\left(\frac{\alpha \beta}{\alpha+\beta}(x+y)\right)
$$

hence it follows that

$$
m\left(\frac{\alpha \beta}{\alpha+\beta}(x+y)\right)=\frac{\beta}{\alpha+\beta} m(\alpha x)+\frac{\alpha}{\alpha+\beta} m(\beta y),
$$

namely, $\alpha x=\beta y$. Therefore, the first norm is strictly convex.

REMARK 1. There are Nakano spaces with strictly convex modulars and its conjugate modulars are not strictly convex. As the simplest example, the Nakano space of real numbers with its modular:

$$
f(\xi)=\left\{\begin{array}{cr}
\frac{1}{2} \xi^{2} & (0 \leqq \xi \leqq 1), \\
2 \xi-\log \xi & (\xi>1)
\end{array}\right.
$$

is strictly convex but not increasing. Therefore, its conjugate modular is not finite, and so, is not strictly convex.

REMARK 2. Even if the norms are strictly convex, the modular is not necessarily strictly convex. As the example, let us take a Orlicz sequence space:

$$
m(x)=\sum_{\nu=1}^{\infty} f\left(\xi_{\nu}\right) \quad \text { for } x=\left(\xi_{1}, \xi_{2} \cdots\right)
$$

where

$$
f(\xi)=\left\{\begin{array}{cll}
\xi^{2} & \text { if } & 0 \leqq \xi \leqq 1 \\
+\infty & \text { if } & \xi>1
\end{array}\right.
$$

This modular is not strictly convex, but

$$
\|x\|=\left(\sum_{\nu=1}^{\infty}\left|\xi_{\nu}\right|^{2}\right)^{1 / 2} .
$$

REFERENCES

\section{Amemiya}

1. A characterization of modulars of $L_{p}-t y p e$ J. Fac. Sci. Hokkaidô Univ. vol. 12 (1953) pp. $22-33$.

S. MAZUR

1. Über konvexe Mengen in linearen normierten Raumen, Studia Math. vol. 4 (1933) pp. 70-84. 
T. Mori, I. Amemiya and H. Nakano

1. On the reflexivity of semi-continuous norms, Proc. Japan Acad. vol. 31 (1955) pp. 684-685. H. NAKANO

1. Modulared semi-crdered linear spaces, Tokyo, 1950.

2. Modern spectral theory, Tokyo, 1950.

3. Modulared linear spaces, J. Fac. Sci. Univ. Tokyo vol. 6 (1951) pp. 85-131.

S. YAMAMURO

1. On linear modulars, Proc. Japan Acad. vol. 27 (1951) pp. 623-624.

2. Exponents of modulared semi-ordered linear spaces, J. Fac. Sci. Hokkaidô Univ. vol. 12 (1953) pp. 211-253.

3. Modulared sequence spaces, ibid. vol. 13 (1954) pp. 13-21.

HoKKaIDô UNIVERSITY,

HOKKAIDô, JAPAN

The Institute for Advanced Study,

Princeton. N. J. 